\title{
XXIV.
}

Aus der Königl. psychiatrischen und Nervenklinik zu Halle (Prof. Hitzig).

\section{Ueber eine Art progressiver Heredität bei Huntington'scher Chorea.}

\author{
Von \\ Prof. Dr. Heilbronner, \\ Oberart der Klinik.
}

Vor Kurzem stellte sich in der hiesigen Poliklinik ein Kranker vor, der einer uns seit Jahren bekannten von Facklam ${ }^{1}$ ) bereits ausführlicher beschriebenen, auch von Wollenberg ${ }^{2}$ ) erwähnten Choreatikerfamilie angehört; er stellt das erste erkrankte Glied der im Uebrigen anscheinend noch frei gebliebenen vierten Generation (von dem choreatischen Grossvater aus gerechnet) dar, ist ein Sohn des einzigen männlichen Gliedes der dritten Generation, des hier lange stationär behandelten und ror Kurzem verstorbenen Ferdinand Z.; die Erkrankung zeigt die typischen Züge der Huntington'schen Chorea: ausgebildete choreatische Bewegungen, sehr intensive Reizbarkeit und eine bereits erhebliche Intelligenzabnahme, zunächst keinerlei klinische Erscheinungen, die von dem geläufigen - in der hiesigen Klinik übrigens doch recht häufig zur Beobachtung kommenden - Bilde der H.'schen Chorea abgewichen wären. Die Erkrankung hat sich seit mehreren Jahren schleichend entwickelt; gegenwärtig ist der Kranke 2o Jahre alt; der Beginn ist also mindestens in die ersten Jahre des 3. Decenniums zu setzen.

Die letztere Thatsache rief mir eine vor einer Reihe von Jahren noch in der Breslauer Klinik gemachte Beobachtung in's Gedächtniss,

1) Facklam, Beiträge zur Lehre vom Wesen der Huntington'schen Chorea. Dieses Archiv Bd. 30. S. 165.

2) Wollenberg, Chorea, Paralysis agit. etc. Wien 1899. S. 79. 
über die mir leider schriftliche Notizen nicht zur Verfügung stehen; die recht auffallenden Verhältnisse batten sich mir aber mit für den vorliegenden Zweck hinreichender Genauigkeit eingeprägt.

Bei der Aufnahme einer ca. 50 jährigen Paranoica wurde mir als einziges hereditäres Moment von der begleitenden Schwester angegeben, dass die Mutter in hohem Alter an einem erst in den Greisenjahren aufgetretenen oder jedenfalls bemerkten Veitstanz gestorben sei. Gelegentlich eines. Besuches constatirte ich daun zu meiner Ueberraschung bei einer weiteren, Ende der 40 er Jahre stehenden Schwester der Patientin eine exquisite Chorea, die, wie mühsam eruirt werden konnte, ebenfalls seit einigen Jahren bestand, und als ich mir nach einiger Zeit das Vertrauen der Familie erworben, wurde mir ein 18 oder 19 jähriges Mädchen, die Tochter einer vierten (angeblich nicht choreatischen) Schwester vorgestellt, bei der sich gleichfalls choreatische Bewegungen seit einiger Zeit ganz schleichend entwickelt hatten und bei der Untersuchung nachweislich waren.

Dass die Huntington'sche Chorea nicht, wie der erste Darsteller der Erkrankung meinte, aussehliesslich in den mittleren Jahren auftritt, ist jetzt wohl allgemein anerkaunt; die Beobachtung einiger ungewöhnlich junger Patienten (nach Wollenberg ${ }^{1}$ ) kommt die Erkrankung vor dem 25. (ebenso wie nach dem 55.) Lebensjahre "nur ausnahmsweise" vor) gäbe also immerhin noch keinen Anlass zur Hittheilung an dieser Stelle. Dagegen drängte sich mir anlässlich der neuen Beobachtung doch wieder die schon früher erwogene Frage auf, ob etwa das Tieferrücken des Anfangsalters der Erkrankung in jeder folgenden Generation, wie sie gerade in dem letztangeführten Beispiel so auffallend zu Tage trat, öfter und einigermassen regelmässig zu beobachten sei.

Allgemeineres Interesse scheint ein solches Verhalten bis jetzt nicht erregt zu haben; in der umfassenden Darstellung Wollenberg's, in der dem auch sonst von den Autoren aufmerksam verfolgten Alter beim Krankheitsbeginn ein besonderer Abschnitt gewidmet ist, ist es jedenfalls nicht erwähnt, und ein kurzer Hinweis auf ein nach mancher Richtung interessantes Verhalten scheint mir deshalb hier berechtigt, selbst für den Fall, dass etwa, was mir entgangen sein mag, schon gelegentlich auf ein derartiges Verhältniss hingewiesen sein sollte.

Ich habe mich, um jeder Versuchung aber auch jedem Verdachte willkürlicher Auswahl der Fälle zu entgehen, zunächst darauf beschränkt, die von Wollenberg mitgetheilten Stammbäume, soweit sie mindestens 3 Generationen unfassen, in der eben bezeichneten Richtung durchzu-

1) l. .. p. 85 . 
Ueber eine Art progressiver Heredität bei Huntington'scher Chorea. 891 prüfen. Das Ergebniss lasse ich wieder in Form der Stammbäume hier folgen (mit Auslassung aller, hier nicht interessirenden, nicht choreatischen Familienglieder).

Familie W. (Zacher).

\begin{tabular}{lll|c}
$\overbrace{\text { W. }+45}^{M . ?}$ & M.? & M.? & ? \\
$\overbrace{\text { W. B. } 41}^{\text {W. Bo. } 12 \text { (Tic facial). }}$ & W.Bo. 47 & M.B0. 42 & 45 \\
&
\end{tabular}

Familie W. Z. (hiesige Klinik).

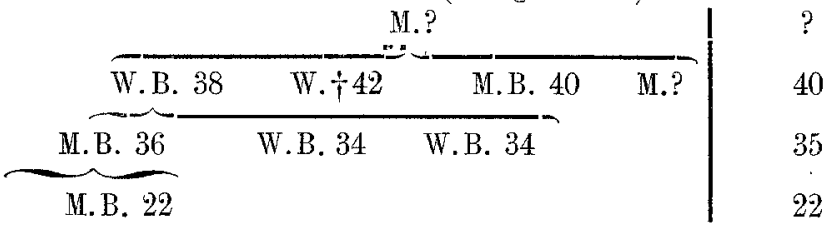

Familie 0. (Ewald).

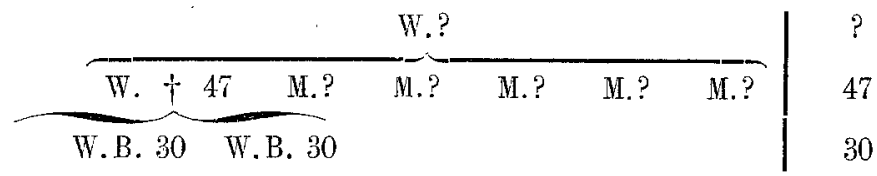

Familie Waldi-Wipfler (Hoffmann).

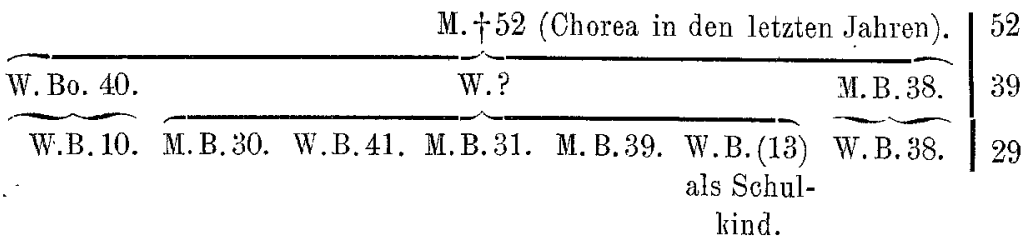

Familie Vey (Lannois).

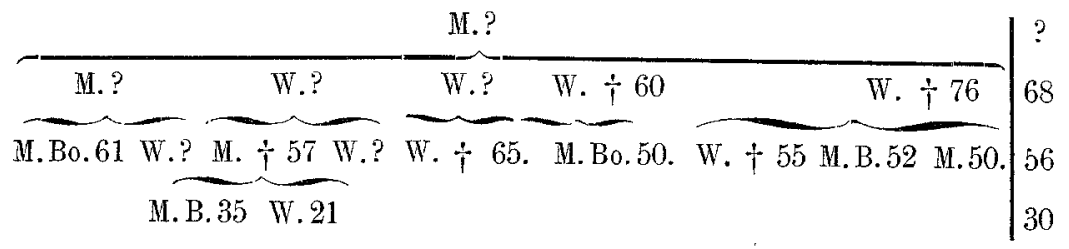

Abkürzungen: M. Mann. W. Weib. ? Altex unbekannt. B. x Beginn mit $x$ Jahren. $\dagger \mathrm{x}$ Tod mit $\mathrm{x} J a h r e n$, Bo. $\mathrm{x}$ Alter zur Zeit der Beobachtung. 
M.? W?

$\mathrm{W}+67$

M. B. 40

M. $\div 60$ M.B. 57 W.B. 54 W.B. 54 W. $\uparrow 40$ M. $\uparrow 45$ M. $\uparrow 42$ W.B. 39 M. $\uparrow 19$ W.B. 32

Q M.?? in der

Sehule.

Um eine wenigstens ungefähre Uebersicht zu gewinnen, habe ich für jede Generation das arithmetische Mittel in gewohnter Weise berechnet; die gewonnenen Zahlen sind jeweils beigesetzt und würden, wenı sie ohne Weiteres als Ausdruck der thatsächlichen Verhältnisse zu erachten wären, die auf Grund der eingangs erwähnten Fälle aufgestellte Vermuthung in sehr evidenter Weise bestätigen. Thatsächlich wird aber die Verwerthbarkeit der Zahlen durch einige Fehlerquellen beeinträchtigt.

Zunächst brachte es die Art des Materiales mit sich, dass die Altersangaben auch innerhalb $j e$ eines Stammbaumes sich auf verschiedene Momente - einmal den des Krankheitsbeginns, einmal des tödtlichen Ausganges, in anderen auf das Alter zur Zeit der Beschreibung: - beziehen und so verrechnet werden mussten; da gerade in den älteren Generationen natürlich häufiger das Alter beim Ableben angegeben ist, so werden dadurch bei der langen Dauer der Erkrankung die Durchschnittszahlen diesen Generationen an sich höher. Dass aber dieses Joment nicht allein die sebr auffallenden Differenzen bedingt, ergiebt sich daraus, dass sie auch nicht schwinden, wemn nur die Falle beriicksichtigt werden, für die das Anfangsalter angegeben ist.

Auch zwei weitere Fehlerquellen, an die ich gedacht, scheinen mir doch nicht ausreichend, die Differenz zu verursachen: es liegt ja nahe, einzuwenden, dass jederzeit von den Gliedern der letzten Generationen noch Angehörige in höherem Lebensalter erkranken und so deren Durchschnittszahl erhöhen können, während anderseits das eine oder andere jung erkrankte und verstorbene Glied früherer Generationen zur Zeit der Nachforschungen vergessen sein mag.

Gerade die letztere Fehlerquelle scheint mir nicht von allzu grosser Bedeutung: die familiäre Erkrankung pflegt, wie man sich des Oefteren $z u$ überzeugen Gelegenheit hat, ein sehr lebhaftes Interesse nicht nur bei den Nächstbetheiligten, sondern oft auch darüber hinaus zu erregen, so sehr, dass mir z. B. bei unserer letztzugeführten Chorea ein Nachbar spontan, ehe ich noch danach zu fragen Zeit hatte, den ganzen - von den Angehörigen dann bestätigten - choreatischen Stammbaum zu ent- 
wickeln vermochte; man erhält also derartige Angaben - einigermassen geordnete sociale Verhältnisse vorausgesetzt - relativ gut und vollständig. Die Thatsache, dass gerade in der letzten Generation öfter Kinder oder Individuen im Beginn des 3. Decenniums als befallen angeführt werden, gewinnt damit erst Werth.

An der Hand dieser Ueberlegungen erst schien es mir zulässig, auch in anderen vereinzelten, im gleichen Sinne sprechenden Mittheilungen mehr als den Ausdruck zufälligen Zusammentreffens zu erblicken; so will ich erwähnen, dass der erste der von Facklam ${ }^{1}$ ) beschriebenen Kranken, dessen Grossvater, Vater und 2 Geschwister choreatisch waren, und der selbst mit ca. 40 Jahren erkrankte, ein 10 jähriges an der gleichen Affection leidendes Töchterchen hatte. Aus der Jenenser Klinik berichtet Goldstein²) über einen Kranken, der 57 jährig nach ca. 18 jähriger Krankheit starb, also ca. 39 jährig erkrankt war; sein Vater war wahrscheinlich choreatisch gewesen; mit dem Kranken zuglejch wurde dessen 26 jährige Tochter wegen chronischer, schon seit 3 bis 4 Jahren bestehender, Chorea behandelt.

Eine Nachuntersuchung der hier besprochenen Verhältnisse auch in den Choreatikerfamilien, die nicht durch die mächtige Verzweigung ihrer Stammbäume imponiren, erscheint jedenfalls angebracht und kann vielleicht noch Aufschluss nach mancher Richtung geben.

Ich bin, wie ich kaum zu betonen branche, weit davon entfernt, etwa behaupten zu wollen, dass die Kinder eines choreatischen Ascendenten, wenn überhaupt, unter allen Umständen in jüngeren Jahren von der Chorea ergriffen werden müssten, als der Ascendent. Eine derartige Annahme liesse sich wohl nur in totaler Verkennung der Art und Weise aufstellen, wie allgemeine Gesetze bei der Vielheit der concurrirenden Homente in die Erscheinung treten können. Soviel aber glaube ich bei aller Skepsis, die einem relativ kleinen Material gegenüber doppelt angebracht erscheint, doch wahrscheinlich gemacht zu haben, dass der familiären Chorea im Allgemeinen die Tendenz beiwohnt, in jeder folgenden Generation im Durchschnitt jüngere Individuen zu befallen als in der vorbergegangenen.

In dieser Tendenz würde sich - wenn weitere Forschungen das Gesagte bestätigen - in besonders instructiver Weise die von Wollenberg betonte degenerative Natur der Huntington'schen Chorea documentiren. Entsprechend der Meinung der Majorität der Autoren wïrde auch ein

1) I. c. S. 154 .

2) Goldstein, Ein Fall von Huntington'scher Chorea. Inaug.-Diss. Jena 1897. 
894 Prof. Dr. Heilbronner, Ueber progr. Her. bei Huntington'scher Chorea.

derartiger, nicht nur in Bezug auf das Individuum, sondern auch auf den Stamm progressiver Verlauf für die Berechtigung der Sonderstellung der Huntington'schen Chorea gegenüber anderen - auch protahirt oder chronisch verlaufenden - Füllen sprechen; er würde weiterhin vielleicht in dem Sinne zu verwerthen sein, dass unbeschadet des relativ häufigen Vorkommens anderer Neurosen und Psychosen in den Choreatikerfamilien die gleichmässige Vererbung im innersten Wesen des krankhaften Processes begründet ist, wie sie ja auch bei wenig anderen Erkrankungen in gleich exquisiter Weise dureh Generationen zu verfolgen ist. Dass wir trotz alledem über das Wesen dieses krankhaften Processes selbst, insbesondere über die Genese und den Auslösungsmodus der choreatischen Bewegungen, so wenig erfahren, wie vorher, möchte ich dabei nicht zu erwähnen unterlassen.

Das Vorrücken des Erkrankungsalters von einer Generation zur andern würde, wie ich noch erwähnen möchte, eine neue, soviel mir bekannt, noch nicht betonte Furm der progressiven Heredität, jener besonders von Horel studirten, im Ganzen früher mit lebhafterem Interesse als hente discutirten Form der Vererbung darstellen. Während Morel bekanntlich mehr eine qualitative Progression eintreten sah, würde hier eine gewissermassen quantitative und bis zu einem gewissen Grade zahlenmässig ausdrückbare Progression zu statuiren sein. Yan wird die Schwere der erblichen Belastung, da wo die Wirkung selbst eine qualitativ gleiche ist, für um so höher zu halten berechtigt sein, je früher sie ihre Wirkung ehen durch den Ausbruch der Erkrankung documentirt.

Es wäre von Interesse festzustellen, ob dem früheren Ausbruch auch ein schwererer Verlauf in den späteren Generationen entspricht, sei es, dass die ganze Krankheitsdauer verkürzt wird, sei es, das sich die Demenz etwa ungewöhulich rasch entwickelt. Ich verkenne nicht, dass derartige Untersuchungen nur mit äusserster Vorsicht anzustellen wären, und dass die ganze Frage ganz ausserordentlich complicirt wird, schon deshalb, weil uns noch ganz unverständlich ist, woher bei dem vorausgesetzten ersterkrankten Ahnen jeder Choreafamilie die Erkrankung und ganz besonders die exquisite Tendenz zur qualitatir gleichförmigen Vererbung stammen mag, und weil wir anch in keinem Falle mit Bestimmtheit den wirklichen Ersterkrankten festzustellen vermögen. Trotzdem scheinen mir gerade die Choreafamilien zur Nachprüfung mancher allgemeiner Fragen der Vererbung besonders geeiguet, eben weil hier bei der cqualitativen Gleichartigkeit der krankhaften Processe quantitative, leichter vergleichbare Verhältnisse reiner zu Tage treten werden. 